\title{
Widely tunable monolithic narrowband grating filter for near-infrared radiation
}

\author{
Frank Brückner, ${ }^{1, *}$ Stefanie Kroker, ${ }^{1}$ Daniel Friedrich, ${ }^{2}$ Ernst-Bernhard Kley, ${ }^{1}$ and Andreas Tünnermann ${ }^{1}$ \\ ${ }^{1}$ Institut für Angewandte Physik, Friedrich-Schiller-Universität Jena, Max-Wien-Platz 1, 07743 Jena, Germany \\ ${ }^{2}$ Max-Planck-Institut für Gravitationsphysik (Albert-Einstein-Institut) and Institut für Gravitationsphysik, \\ Leibniz Universität Hannover, Callinstrasse 38, 30167 Hannover, Germany \\ *Corresponding author: frank.brueckner@uni-jena.de
}

Received December 2, 2010; revised December 24, 2010; accepted December 24, 2010; posted January 10, 2011 (Doc. ID 139086); published January 31, 2011

\begin{abstract}
We propose a monolithic narrowband guided-mode grating filter in fused silica that is widely tunable in the near-IR wavelength region. Based on a recently demonstrated approach for a monolithic reflector comprising an encapsulated grating, we theoretically investigate such a device by means of rigorous modeling aimed at a narrow linewidth. It is demonstrated that upon a spatial variation of the filter's grating period its resonance wavelength can be tuned in a remarkably wide range of near-IR radiation with $800 \mathrm{~nm}<\lambda_{\text {res }}<1600 \mathrm{~nm}$ by translating the laser beam relative to the grating area. The filter performance in terms of linewidth and contrast is essentially preserved over the entire tuning interval. (c) 2011 Optical Society of America

OCIS codes: $\quad 050.1950,050.6624,230.3990,230.7408$.
\end{abstract}

The guided-mode resonance phenomenon of diffraction gratings is a type of grating anomaly observed in subwavelength structures of thin-film waveguide layers [1]. When precise phase-matching conditions are met, externally propagating waves can be coupled into guided modes by the grating. A refractive index modulation in the waveguide layer causes the guided modes to slowly leak from the waveguide and to recombine with the incident wave to establish a resonance [2]. Such leaky modes generated in periodically corrugated waveguide layers are capable of a variety of applications such as narrowband reflection and transmission filters [3], tunable filters [4], broadband laser cavity reflectors [5,6], optical switch devices [7], and light modulators [8]. Recently, resonant gratings have also been proposed for use in the field of high-precision interferometry serving as low thermal noise optical components due to their thin-film layout, which minimizes the thickness of the necessary dielectric coating [9]. Very recently, we demonstrated two approaches for purely monolithic guided-mode surface reflectors that avoid any dielectric coating at all $[\underline{6}, \underline{10}$ ], one of which comprises an encapsulated grating with a flat and robust surface. Such a reflector as shown in Fig. 1(a) inherently provides a weak perturbation of the waveguide layer that gives a small diffraction efficiency into the waveguide modes and thus a narrow resonance spectral bandwidth [2]. The narrowing is further supported when using fused silica owing to its very low refractive index contrast to air $(\Delta n \approx 0.5)$ [2]. Hence, such a fused-silica encapsulated grating would not be suitable for broadband applications as intended by our previous work, but it does represent a new type of a monolithic narrowband filter whose spectral reflectivity linewidth can be expected to be in a range of only a few nanom-

eters. Since fused silica is widely transparent from UV to near-IR (NIR), the working wavelength can be freely defined in this range by choosing appropriate grating parameters such as the period $d$ or the waveguide thickness $s$. Consequently, a linear spatial variation of one of these parameters over a given grating length should offer a tu- nabilty of the resonance peak over a wide spectral range by simply translating the laser beam relative to the grating [4]. In the following we will theoretically investigate such a new type of a narrowband tunable filter working in a wide NIR band ( $800 \mathrm{~nm}<\lambda_{\text {res }}<1600 \mathrm{~nm}$ ) by means of rigorous simulation and discuss relevant issues for a fabrication process.

For our design considerations, we assume the grating period to be the spatially varying parameter while the waveguide layer thickness remains constant. Such a configuration seems more suitable for state-of-the-art fabrication techniques, since a variation of the grating period can be accurately controlled during electron-beam lithography, whereas a variation of the cover layer thickness would be much more difficult in practice and should be chosen constant such as realized in [10] for silicon. Figure 1(a) shows the basic idea of our proposed monolithic narrowband tunable filter where the resonance peak position is controlled via the grating period ranging from $d_{\min }$ to $d_{\max }$. The tuning of the narrowband reflected wavelength peak between $\lambda_{\min }$ and $\lambda_{\text {max }}$ can thus be realized by translating the laser beam relative to the grating or vice versa. For a fused-silica encapsulated grating with fixed parameters such as a period of $d=797 \mathrm{~nm}$, a cover layer thickness of $s=700 \mathrm{~nm}$, a groove depth of $g=$ $1500 \mathrm{~nm}$, and a fill factor of $f=r / d=0.4$ (assuming TM-polarized light under normal incidence), Fig. 1(b) shows its resonance peak (black curve) which is in this case located at $\lambda_{\text {res }}=1064 \mathrm{~nm}$ with an FWHM of only $1 \mathrm{~nm}$. Such a small linewidth is essential for filter applications [2,3]. With regard to a nonvanishing angular divergence of real collimated beams of typically $\Delta \phi=$ $1 \mathrm{mrad}$ [11], we also plotted the filter's angular tolerance at $\lambda_{\text {res }}=1064 \mathrm{~nm}$ (red curve) with a resulting FHWM of $1 \mathrm{mrad}$. Hence, the usage of real beams will not significantly affect the filter efficiency. Moreover, the contrast between resonance and off-resonance reflectivity exceeds a value of 20 , which should be sufficient for most experiments. Aiming at a tunable wavelength range in the NIR region with $\lambda_{\text {min }} \approx 800 \mathrm{~nm}<\lambda_{\text {res }}<\lambda_{\text {max }} \approx 1600 \mathrm{~nm}$, including prominent laser emission spectra around 


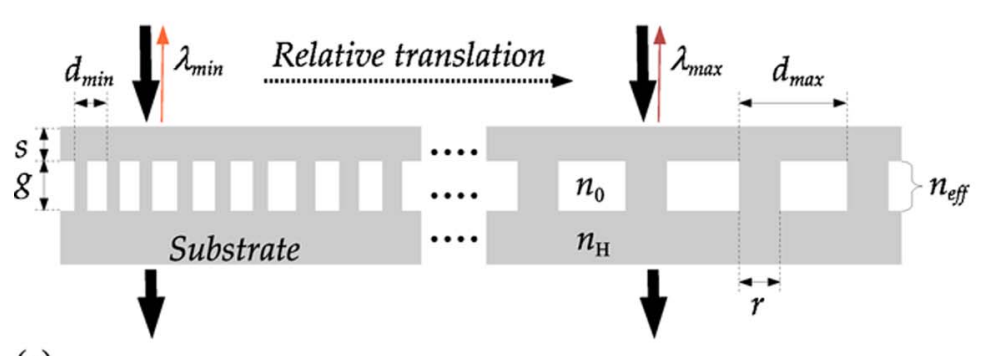

(a)

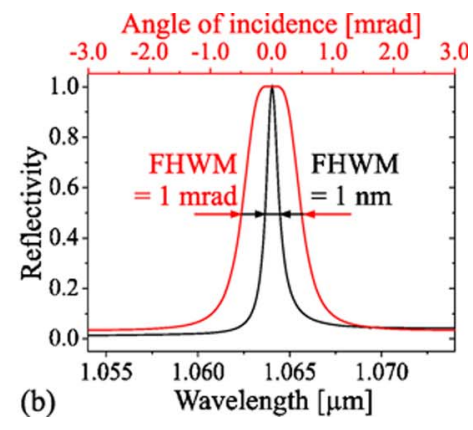

Fig. 1. (Color online) (a) Encapsulated grating with varying period as a monolithic narrowband tunable filter, (b) resonance peak at $\lambda=1064 \mathrm{~nm}$ (black curve) and its angular tolerance (red curve) with an FWHM of $1 \mathrm{~nm}$ and 1 mrad, respectively, for a fused-silica encapsulated grating with $d=797 \mathrm{~nm}, s=700 \mathrm{~nm}, g=1500 \mathrm{~nm}$, and $f=0.4$ (TM-Pol.).

$800 \mathrm{~nm}$ (Ti:sapphire), $1030 \mathrm{~nm} \quad$ (Yb:YAG), $1064 \mathrm{~nm}$ (Nd:YAG), and $1550 \mathrm{~nm}$ (InGaAsP), a grating period variation roughly ranging from $d_{\min }=550 \mathrm{~nm}$ to $d_{\max }=$ $1600 \mathrm{~nm}$ has to be considered. This range is determined by the following inequality (derived from the well-known grating equation), which needs to be held to allow for resonant reflection in principle [2]:

$$
\lambda / n_{H}<d<\lambda / n_{\mathrm{eff}},
$$

with $n_{H} \approx 1.45$ for fused silica (accounted for dispersion) and $n_{\text {eff }} \rightarrow 1$, the effective low refractive index of the grating layer as already used in [10]. By means of rigorous simulation [12], the reflectivity from a fused-silica encapsulated grating was calculated for a simultaneous variation of the wavelength and the grating period ranging from 780 to $1600 \mathrm{~nm}$ and 570 to $1400 \mathrm{~nm}$, respectively and is plotted in Fig. 2. For this calculation, a constant cover layer thickness of $s=700 \mathrm{~nm}$, a groove depth of $g=1500 \mathrm{~nm}$, a fill factor of $f=0.4$, and TMpolarized light impinging under normal incidence had been assumed. As desired, a single resonance peak moves almost linearly through the entire NIR wavelength region upon the grating period variation as chosen above. The found wavelength shift of the resonance peak $\Delta \lambda[\mathrm{nm}]$ is almost equal to the change in period $\Delta d[\mathrm{~nm}]$.

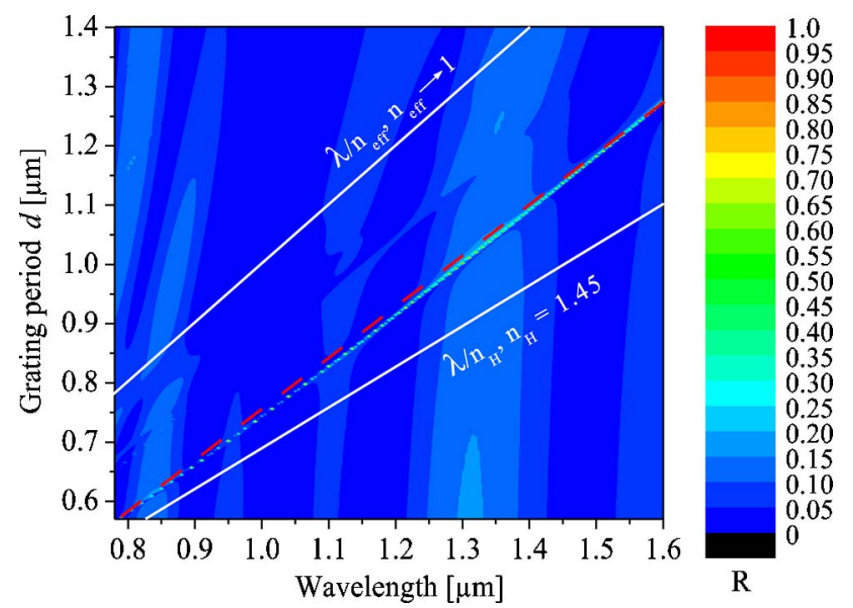

Fig. 2. (Color online) Reflectivity against the wavelength $\lambda$ and the period $d$ of a fused-silica encapsulated grating with $s=700 \mathrm{~nm}, g=1500 \mathrm{~nm}$, and $f=0.4$, assuming TM-polarized light under normal incidence. The resonance line is interrupted due to the discretization of the parameter grid. Dashed red line, linear fit according to Eq. (2).
Thus, the dependency of the resonance wavelength $\lambda_{\text {res }}$ on the grating period $d$ can be well approximated by the linear function (dashed red line):

$$
\lambda_{\text {res }}[\mathrm{nm}]=1.15 \cdot d[\mathrm{~nm}]+135 \mathrm{~nm} .
$$

The white lines indicate the boundaries of the parameter region where resonant reflection is possible for a given wavelength, according to Eq. (1) and assuming an effective index approaching $n_{\text {eff }} \rightarrow 1$. Please note that the resonance line in Fig. 2 appears interrupted only due to the discretization of the parameter grid. To show its continuity in peak reflectivity and linewidth over the entire tuning interval, Figs. 3(a)-3(c) plot the resonance peaks for a slight variation around three very prominent laser wavelengths: (a) $800 \mathrm{~nm}$, (b) $1060 \mathrm{~nm}$, and (c) $1550 \mathrm{~nm}$. Even though the resonance linewidths as well as the ratios between resonance and off-resonance reflectivity are not perfectly constant throughout the entire tuning interval, such a filter performance with a wide spectral range of $d_{\max }-d_{\min }=800 \mathrm{~nm}$ has to the best of our knowledge not been reported before including nonmonolithic grating layouts. An expansion of the tuning interval to even shorter or larger wavelengths without affecting the filter performance is unfortunately not possible. This is because for shorter wavelengths a second waveguide mode arises in the waveguide layer leading to a second resonance peak, while for larger wavelengths the mode matching becomes worse resulting in a decreased peak reflectivity. This is attributed to the choice of the thickness $s$ of the waveguide layer, which is able to support the propagation of a single waveguide mode only for a limited wavelength range. Our waveguide layer thickness of $s=700 \mathrm{~nm}$ was hence not chosen arbitrarily but rather for an optimum performance in the particular NIR region targeted.

For fabrication of our fused-silica tunable filter, we propose to employ the same approach as realized for the encapsulated grating fabricated from silicon in [10]. After etching a conventional surface relief grating into a fused-silica substrate, a thin and flat surface cover layer can be subsequently obtained either by applying wafer direct bonding techniques or by a controlled material deposition without filling the grating grooves followed by a final CMP step (chemical-mechanical polishing). All fabrication techniques potentially involved such as wafer bonding or CMP are suitable for fused-silica processing $[\underline{13}, \underline{14}]$. The desired grating period variation that enables 

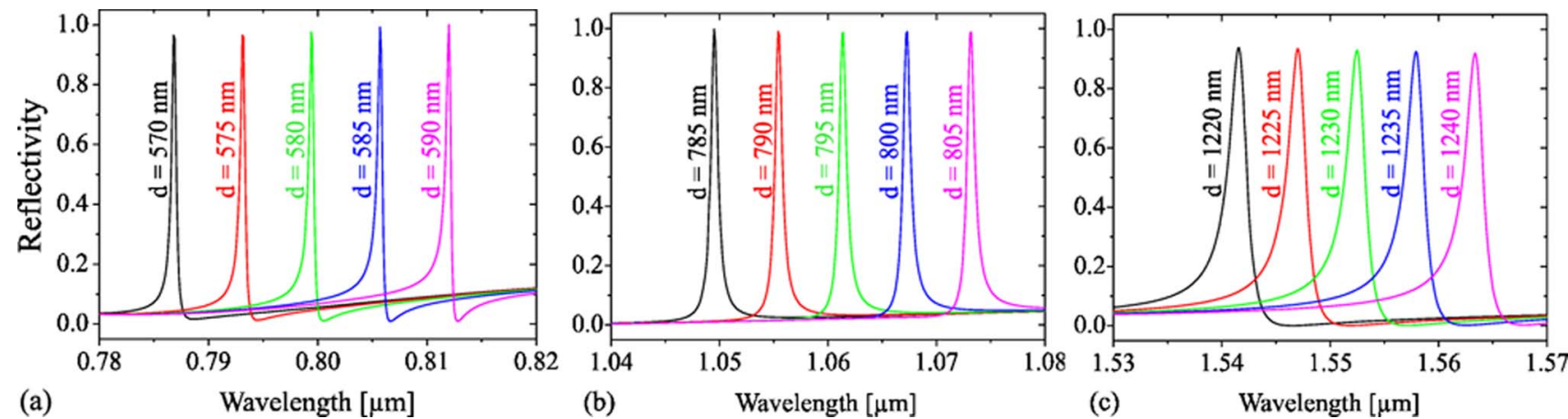

Fig. 3. (Color online) Tuning of the spectral reflectivity resonance peak around frequently used NIR wavelengths of (a) $800 \mathrm{~nm}$, (b) $1060 \mathrm{~nm}$, and (c) $1550 \mathrm{~nm}$ by varying the grating period in an appropriate range.

the tuning of the resonance peak is accessible by state-ofthe-art electron-beam lithography, which is part of the surface relief grating fabrication. An electron-beam facility such as the Vistec SB350 OS allows one to adjust the period with an increment of $1 \mathrm{~nm}$ during each exposure step. In our case, this corresponds to a fundamental minimum shift of the resonance peak of about $1 \mathrm{~nm}$ as well, according to Eq. (2). For the entire wavelength range from 800 to $1600 \mathrm{~nm}$, a period variation between 580 and $1275 \mathrm{~nm}$ is necessary (see Fig. 2), which for a desired minimum shift of $1 \mathrm{~nm}$ would cause 695 variation steps of the grating period during electron beam exposure. The overall required grating length will finally be defined by the laser beam radius, since the beam must impinge on a grating spot having only one particular period to gain the theoretically predicted narrow linewidth. In this regard, also the lateral propagation length of the leaky modes needs to be considered, which is in the range of approximately $0.2 \mathrm{~mm}$ for our filter [11]. For instance, a laser beam diameter of $1 \mathrm{~mm}$ and an amount of 695 variation steps result in a minimum grating length of $69.5 \mathrm{~cm}$. Of course, such a wide grating must be split into subgratings in order to be patterned onto a single fused-silica substrate. In summary, both the available laser beam size and the desired increment of the resonance wavelength shift determine the necessary grating dimensions in practice.

This Letter is supported by the Deutsche Forschungsgemeinschaft within the Sonderforschungsbereich TR7.

\section{References}

1. E. Popov, L. Mashev, and D. Maystre, Opt. Acta 33, 607 (1986).

2. A. Sharon, D. Rosenblatt, and A. A. Friesem, J. Opt. Soc. Am. A 14, 2985 (1997).

3. R. Magnusson and S. S. Wang, Appl. Phys. Lett. 61, 1022 (1992).

4. J.-S. Ye, Y. Kanamori, F.-R. Hu, and K. Hane, J. Mod. Opt. 54, 827 (2007).

5. M. C. Y. Huang, Y. Zhou, and C. J. Chang-Hasnain, Nat. Photon. 1, 119 (2007).

6. F. Brückner, D. Friedrich, T. Clausnitzer, M. Britzger, O. Burmeister, K. Danzmann, E.-B. Kley, A. Tünnermann, and R. Schnabel, Phys. Rev. Lett. 104, 163903 (2010).

7. A. Mizutani, H. Kikuta, and K. Iwata, J. Opt. Soc. Am. A 22, 355 (2005).

8. T. Katchalski, G. Levy-Yurista, A. Friesem, G. Martin, R. Hierle, and J. Zyss, Opt. Express 13, 4645 (2005).

9. A. Bunkowski, O. Burmeister, D. Friedrich, K. Danzmann, and R. Schnabel, Classical Quantum Gravity 23, 7297 (2006).

10. F. Brückner, D. Friedrich, M. Britzger, T. Clausnitzer, O. Burmeister, E.-B. Kley, K. Danzmann, A. Tünnermann, and Roman Schnabel, Opt. Express 17, 24334 (2009).

11. F. Lemarchand, A. Sentenac, and H. Giovannini, Opt. Lett. 23, 1149 (1998).

12. M. G. Moharam and T. K. Gaylord, J. Opt. Soc. Am. A 71, 811 (1981).

13. B. Olbrechts, X. Zhang, Y. Bertholet, T. Pardoen, and J.-P. Raskin, Microsyst. Technol. 12, 383 (2006).

14. S. T. S. Bukkapatnam, P. K. Rao, W.-C. Lih, N. Chandrasekaran, and R. Komanduri, Appl. Phys. A 88, 785 (2007). 